\title{
Pruebas de ingreso como predictores del rendimiento académico en los aspirantes a soldados del Ejército
}

\author{
Entrance Tests as Predictors of Academic Performance in Army Soldier Applicants \\ GERMÁN GEOVANNY MUÑOZ GUALÁN \\ Pontificia Universidad Católica del Ecuador, Ecuador
}

\section{EFRAIN EDUARDO ZAMBRANO ROSALES}

Pontificia Universidad Católica del Ecuador, Ecuador

RESUMEN: El rendimiento académico del aspirante a soldado es el conjunto de indicadores de materias académicas, preparación física y actitud militar que presenta dentro del instituto de formación militar, puesto que el producto que se pretende entregar al Ejército y la sociedad, es un individuo disciplinado y preparado académica, militar y físicamente, acorde a los estándares establecidos en el Modelo de Educación Militar de Fuerzas Armadas y el plan de carrera en ciencias militares de la Universidad de Fuerzas Armadas - ESPE. Además, es necesario complementar esta formación al inculcar la convicción de hacer una carrera militar profesional y ética. Es así, que el objetivo planteado fue establecer los resultados de las pruebas de ingreso que predicen el rendimiento académico de los aspirantes a soldados de segundo año militar, promoción 2018-2020, de la Escuela de Formación de Soldados del Ejército Ecuatoriano "Vencedores del Cenepa". Por lo cual, esta investigación presenta un enfoque cuantitativo, con diseño no experimental, de tipo transversal y un alcance correlacional. Se utilizó los promedios de las pruebas de ingreso y rendimiento académico por períodos académicos y corte anual de cada aspirante a soldado, del archivo y base de datos de la Escuela de Formación de Soldados "Vencedores del Cenepa", para su tabulación en una matriz de Excel prediseñada y el posterior análisis estadístico en el programa SPSS. De modo que se observa una correlación significativa moderada, entre las pruebas académicas y físicas de ingreso con el rendimiento académico, una correlación baja con la actitud militar de los aspirantes a soldados y la no existencia de correlación con la deserción estudiantil. Además, las pruebas de ingreso que predicen de forma moderada el rendimiento académico y de forma baja la actitud militar de los aspirantes a soldados son las pruebas académicas y físicas de ingreso.

Palabras Clave: Rendimiento académico, Aspirante a soldado, Pruebas de ingreso, Ejército, Ciencias militares. 
ABSTRACT: The academic performance of the aspiring soldier is the set of indicators of academic subjects, physical preparation and military attitude that he presents within the military training institute, since the product that is intended to be delivered to the Army and society is a disciplined and prepared individual academically, militarily and physically, according to the standards established in the Military Education Model of the Armed Forces and the career plan in military sciences of the University of the Armed Forces - ESPE. In addition, it is necessary to complement this training by instilling the conviction of pursuing a professional and ethical military career. Thus, the proposed objective was to establish the results of the entrance tests that predict the academic performance of aspiring second-year military soldiers, 2018-2020 class, of the Ecuadorian Army Soldiers Training School "Vencedores del Cenepa". Therefore, this research presents a quantitative approach, with a non-experimental, cross-sectional design and a correlational scope. The averages of the entrance and academic performance tests were used for academic periods and annual cut-off of each aspiring soldier, from the file and database of the Soldiers Training School "Vencedores del Cenepa", for their tabulation in a matrix of Excel predesigned and the subsequent statistical analysis in the SPSS program. Thus, a moderate significant correlation is observed between academic and physical entrance tests with academic performance, a low correlation with the military attitude of would-be soldiers, and no correlation with student desertion. In addition, the entrance tests that moderately predict academic performance and poorly predict military attitudes of would-be soldiers are the academic and physical entrance tests.

KEYWORDS: Academic performance, Soldier applicants, Entrance exams, Army, Military science.

Recibido: 22 de septiembre de 2020. Aceptado: 30 de octubre de 2020.

Revista de Estudios en Seguridad Internacional, Vol. 6, No. 2, (2020), pp. 117-136. http://www.seguridadinternacional.es/revista/

ISSN: 2444-6157. DOI: http://dx.doi.org/10.18847/1.12.7 


\section{INTRODUCCIÓN}

Se considera al rendimiento académico como el indicador del nivel de aprendizaje que ha alcanzado el estudiante al final de un proceso educativo (Maquilón y Hernández, 2011), lo cual constituye el punto de referencia para valorar el grado de eficacia del proceso de enseñanza-aprendizaje (Santos, García y Vallelado, 2010).

Bajo el concepto de que la educación es la base fundamental e indispensable para el desarrollo de la humanidad (Batallas, 2014), se entiende que la universidad pretende lograr que sus estudiantes, al terminar sus planes curriculares, sean capaces de desenvolverse eficazmente al enfrentarse a un problema en particular, así como participar activamente en el desarrollo y crecimiento de la sociedad.

En Latinoamérica, se evidenció que no existía una única estructura de determinantes del rendimiento académico común entre los países (Fernández, 2004). Por lo que cada región y cada universidad deben procurar identificar los determinantes del rendimiento académico de sus estudiantes, para así elaborar políticas educativas acordes a sus particularidades.

El rendimiento académico es definido por Álvarez y Vallelado (2013) como la productividad del sujeto, es decir el producto final de la aplicación de su esfuerzo, establecido por las actividades, rasgos y percepción más o menos correctos de los conocimientos brindados. Lo cual es el resultado de un conjunto de factores que intervienen en su desarrollo, entre los que pueden encontrarse: a) factores individuales, los que el estudiante no puede modificar, b) factores que pertenecen al alumno, que sí los puede cambiar, y c) factores que están vinculados al contexto educativo, por tanto, escapan del ámbito de influencia del alumno y afectan por igual a todos en el grupo (Álvarez y Vallelado, 2013).

Así mismo se puede clasificar al rendimiento en: a) conceptual, que vincula la valoración de los contenidos conceptuales adquiridos por el alumno, b) procedimental, que se refiere a la capacidad del alumno para ejecutar y resolver problemas relacionados con la materia, y c) actitudinal, que se basa en las intervenciones del alumno en actividades voluntarias propuestas en el desarrollo de la materia (Álvarez y Vallelado, 2013).

En el ámbito militar, el tener presente los factores determinantes del rendimiento académico de los estudiantes es de vital importancia, por toda la gran carga logística que se requiere para el desarrollo de la educación, desde la selección para el ingreso a los institutos de formación, como el entrenamiento y capacitación de los mismos, ya sea por el costo al ser un régimen de internado así como el gasto derivado de la utilización de armamento militar, además de implicar un riesgo para la propia vida como de la población civil un desempeño deficiente (Castro y Fernández, 2011).

Los actuales escenarios operativos, que cada vez son más tecnológicos y caminan paralelamente a la globalización, hacen que los institutos militares a nivel internacional implementen procesos de selección cada vez más rigurosos, lo que requiere que las características de los futuros soldados sean altamente capacitados, adaptables, ingeniosos y rápidos para ajustarse a situación cambiantes y den solución adecuada con la presión social respectiva (Depaula, 2012).

Las Escuelas de Formación Militar, difieren de las Universidades regulares, por las características propias de la educación militar, en las que los jóvenes tienen que realizar dos tareas combinadas durante su formación, por una parte, un intenso entrenamiento militar, que 
trae consigo un constante reto físico, y concomitantemente, una rutina académica universitaria que implica esfuerzo, dedicación y horas de estudio, cuyo resultado final es el logro de un título de tercer nivel y un grado militar (Castro y Fernández, 2011).

Según un gran número de autores, dentro de todos los determinantes existentes, la nota obtenida en las pruebas de admisión a la universidad junto con el rendimiento académico previo a la universidad es el predictor más importante en el rendimiento académico del estudiante (Garbanzo, 2007). Si esta caracterización es rigurosa y objetivamente medible, la selección se limita a ordenar de forma decreciente a los aspirantes y establecer un punto de quiebre para la admisión en cuanto se haya cumplido el cupo establecido (Rodríguez et al, 2000).

El proceso de admisión de forma general se compone de una evaluación de los conocimientos previos de los estudiantes, siendo lo más usual la implementación de instrumentos que valoran habilidades verbales, lógicas, matemáticas y de redacción (Zwick, 2012). En Ecuador el proceso de selección para ingreso a las universidades está determinado al puntaje desarrollado en la evaluación estandarizada denominada prueba ENES (examen nacional de educación superior), así como la oferta establecida por cada universidad (Villacís, 2017). Sin embargo, para el ingreso a las Escuelas de Formación Militar, por ser una educación de riesgo que demanda una máxima exigencia académica, física y mental, es necesario complementar este proceso con una evaluación de habilidades físicas, aptitud médica y psicológicas.

Actualmente se evidencia un incremento en la demanda de ingreso a las escuelas militares de formación. Al tener este escenario de masificación es imperante analizar las pruebas de ingreso establecidas como criterios del proceso de selección, el cual tiene como fin tener estudiantes capacitados para resolver sus tareas académicas de forma óptima y eficiente, así como tener un porcentaje bajo de deserción estudiantil (León, 2009).

Según el informe de gestión institucional del 2015, la Dirección de Personal de la Fuerza Terrestre del Ecuador es quien determina la demanda de aspirantes a ingresar a la Carrera de Ciencias Militares de la Fuerza Terrestre. Una vez determinada la demanda institucional se realiza la convocatoria a nivel nacional de acuerdo a la reglamentación vigente (Universidad de Fuerzas Armadas - ESPE, 2017).

El perfil de ingreso de la carrera de Tecnología en Ciencias Militares es un conjunto de actitudes y aptitudes específicas que debe traer consigo el aspirante a soldado, entre las principales se encuentran: a) compromiso hacia la práctica de principios y valores, b) vocación hacia la vida y servicio militar, c) aptitud e idoneidad física, d) condiciones médicas favorables a la carrera, e) estabilidad y control psico - emocional y f) habilidades y destrezas para trabajar en equipo (Universidad de Fuerzas Armadas - ESPE, 2017).

Las pruebas de ingreso para las Escuelas de Formación Militar están determinadas en cuatro grupos bien diferenciados, mismas que buscan seleccionar a los mejores aspirantes que cumplan tanto el perfil de ingreso como el cupo preestablecido, según detalle: a) pruebas psicológicas, b) pruebas académicas, c) pruebas médicas y, d) pruebas físicas, las cuales se especifican a continuación (COMACO, 2016):

a) Prueba psicológica: valorado por rangos, los cuales van desde rango 1 (A-A), como idóneo, hasta rango 9 (B2-B2), como no idóneo, según detalle: 
-Capacidad mental: valora la inteligencia teórica, inteligencia práctica, aptitud numérica, razonamiento abstracto, aptitud espacial, aptitud verbal, fluidez verbal, aptitud mecánica, aptitud administrativa e inteligencias múltiples.

La calificación del test psicológico de capacidad mental se realiza en base a la escala "C" de Guilford, en donde se clasifica a los aspirantes en: A) recomendable, considerándose como idóneo con capacidades mentales ideales, con un riesgo de fracaso mínimo, B1) no recomendable, primera posibilidad de ser considerado, en el que sus capacidades mentales están cercanas a lo ideal, hay un ligero riesgo de fracaso, B2) no recomendable, segunda posibilidad de ser considerado, en el que sus capacidades mentales son intermedias, hay un mediano riesgo de fracaso, B3) no recomendable, tercera posibilidad de ser considerado, en el que sus capacidades mentales están alejadas de lo ideal, hay un alto riesgo de fracaso, y C) no recomendable, sus capacidades mentales son inadecuadas, el riesgo de fracaso es absoluto (CEDE, 2011).

-Test de personalidad: valora los rasgos personales, rasgos familiares, sociales, tipologías y liderazgo.

La calificación de la condición de personalidad, está determinado según los siguientes criterios: A) recomendable, se considera como idóneo, su ajuste de personalidad es el ideal, el riesgo de fracaso mínimo, B1) no recomendable, primera posibilidad de ser considerado, su ajuste de personalidad es cercano a lo ideal, hay un ligero riesgo de fracaso, B2) no recomendable, segunda posibilidad de ser considerado, su ajuste de personalidad es intermedio, hay un mediano riesgo de fracaso, B3) no recomendable, tercera posibilidad de ser considerado, su ajuste de personalidad están alejadas de lo ideal, hay un alto riesgo de fracaso, y C) no recomendable, su ajuste de personalidad es inadecuado, el riesgo de fracaso es absoluto (CEDE, 2011).

b) Prueba académica: valora el conocimiento oral y escrito, matemáticas, realidad nacional, computación e inglés, mismo que es valorado de forma cuantitativa en donde 2019 puntos es excelente, 18,99-18 puntos es muy bueno, 17,99-16 es bueno, 15,99-14 es regular y $<13,9$ es deficiente.

c) Prueba física: valorado cuantitativamente sobre 20 puntos, según una tabla de baremos en donde intervienen las siguientes variables:

-Flexiones de codo: 45 flexiones en 1,30 minutos.

-Flexiones de abdomen: 50 abdominales en 1,30 minutos.

-Trote: distancia de 2 millas en 12,57 minutos.

-Natación: 200 metros en 5,13 minutos.

-Salto de decisión: desde el tablón de 5 metros de altura.

El rendimiento académico de los aspirantes a soldados es un conjunto de indicadores de materias académicas, rendimiento físico, adaptabilidad disciplinaria y espíritu militar que presenta el aspirante dentro de la Escuela de Formación de Soldados del Ejército "Vencedores del Cenepa" durante el desarrollo de la carrera, puesto que el producto que se pretende entregar al Ejército y la sociedad, es un individuo disciplinado, preparado académica, militar y físicamente acorde a los estándares establecidos, además es necesario complementar esta formación inculcando una vida militar profesional y ética. 
El diseño curricular de la Escuela de Formación de Soldados del Ejército "Vencedores del Cenepa" obedece a los lineamientos de la Ley Orgánica de Educación Superior, de la Fuerza Terrestre y de la Universidad de FF.AA - ESPE, en la cual la característica primordial es presentar un modelo educativo dual, en donde por un lado se desarrolla el aprendizaje teórico práctico como tecnólogos superiores en ciencias militares por parte de la Universidad de Fuerzas Armadas - ESPE, así como la formación de habilidades y destrezas como soldados del Ejército por parte de FF.AA. Este currículo está planificado en 5 períodos académicos, con corte anual, en donde el primer año es formativo y el segundo año es de especialidad (Universidad de Fuerzas Armadas - ESPE, 2017), mismos se detallan a continuación:

I período académico: instrucción individual de combate, legislación militar, navegación terrestre, cultura física.

II período académico: escuadra de fusileros, conocimiento de armas de fuego, inglés A1, fundamentos de matemáticas, comunicación oral y escrita, y cultura física.

III período académico: técnicas y tácticas de patrullaje, inglés A2, liderazgo, metodología de investigación científica, derecho aplicado a las operaciones y cultura física.

IV período académico: diseño de proyectos, inglés B1, operaciones militares, historia militar, realidad nacional y geopolítica, administración general, organización de armas y servicios, fase de selva y cultura física.

V período académico: teoría de la docencia, cultura ambiental, fase de especialidad, producto integrador y cultura física.

El objetivo general planeado del presente trabajo investigativo es establecer qué resultados de las pruebas de ingreso predicen el rendimiento académico de los aspirantes a soldados, promoción 2018-2020, de la Escuela de Formación de Soldados del Ejército "Vencedores del Cenepa". Para lo cual es imprescindible cumplir con los siguientes objetivos específicos: a) detallar los resultados de las pruebas de ingreso psicológicas, académicas y físicas que presentaron los aspirantes a soldados, b) enunciar los resultados del rendimiento académico, así como deserción estudiantil y actitud militar por períodos académicos y con corte anual de los aspirantes a soldados, c) comparar la relación existente entre los resultados de las pruebas de ingreso psicológicas, académicas y físicas, con el rendimiento académico, así como con la deserción estudiantil y actitud militar de los aspirantes a soldados, y d) encontrar el resultado de las pruebas de ingreso psicológicas, académicas y físicas óptimas para predecir el rendimiento académico, así como la deserción estudiantil y actitud militar de los aspirantes a soldados.

La hipótesis planteada del presente trabajo es: Los resultados de las pruebas de ingreso a las Escuelas de Formación Militar se relacionan directamente en el rendimiento académico de los aspirantes a soldados, promoción 2018 - 2020, de la Escuela de Formación de Soldados del Ejército "Vencedores del Cenepa".

El conocer las pruebas de ingreso que se involucran en el rendimiento académico permitiría identificar las variables que determinan el éxito o fracaso del aspirante a soldado, e incluso dejar una guía para que la comisión de selección de cada proceso de reclutamiento en los próximos años tome sus decisiones con el conocimiento de los posibles efectos a futuro. 
Situación por la cual el problema científico se lo ha planteado como la pregunta: ¿Cuáles son las pruebas de ingreso que predicen el rendimiento académico en los aspirantes a soldado, promoción 2018-2020, de la Escuela de Formación de Soldados del Ejército "Vencedores del Cenepa?

\section{MATERIALES Y MÉTODO}

El presente trabajo investigativo es un estudio analítico, que presenta un enfoque cuantitativo, con diseño no experimental, de tipo transversal y un alcance correlacional.

El universo del presente estudio está dado por 1100 aspirantes a soldados de la Escuela de Formación de Soldados del Ejército "Vencedores del Cenepa", siendo la muestra el total de aspirantes de segundo año militar ( $n=619$ aspirantes a soldados), considerado un 3\% de margen de error y un $95 \%$ de nivel de confianza.

El propósito de esta investigación es conocer las pruebas de ingreso psicológicas, académicas y físicas que predicen el rendimiento académico, la deserción estudiantil y la actitud militar de los aspirantes a soldados, promoción 2018 - 2020, de la Escuela de Formación de Soldados del Ejército "Vencedores del Cenepa".

La primera fase, busca detallar los resultados de las pruebas de ingreso psicológicas, académicas y físicas que presentaron los aspirantes a soldados, promoción 2018-2020, en el período de reclutamiento, el cual se desarrolló de abril a julio del 2018, mediante la recolección de información del departamento académico de la Escuela, según detalle:

a) Prueba psicológica: valorado por rangos, los cuales van desde rango 1 (A-A), como idóneo, hasta rango 9 (B2-B2), como no idóneo, según detalle:

-Capacidad mental: A) recomendable, B1) no recomendable, primera posibilidad de ser considerado, B2) no recomendable, segunda posibilidad de ser considerado, B3) no recomendable, tercera posibilidad de ser considerado y $\mathrm{C}$ ) no recomendable.

-Test de personalidad: A) recomendable, B1) no recomendable, primera posibilidad de ser considerado, B2) no recomendable, segunda posibilidad de ser considerado, B3) no recomendable, tercera posibilidad de ser considerado y $\mathrm{C}$ ) no recomendable.

b) Prueba académicas valoración cuantitativa: 20-19 puntos es excelente, 18,99-18 puntos es muy bueno, 17,99-16 es bueno, 15,99-14 es regular y <13,9 es deficiente.

c) Prueba física: valorado cuantitativamente sobre 20 puntos, según una tabla de baremos preestablecida, según detalle:

-Flexiones de codo: 45 flexiones en 1,30 minutos.

-Flexiones de abdomen: 50 abdominales en 1,30 minutos.

-Trote: distancia de 2 millas en 12,57 minutos.

-Natación: 200 metros en 5,13 minutos.

-Salto de decisión: desde el tablón de 5 metros de altura.

Así mismo, se enuncia los resultados del rendimiento académico, por períodos académicos de los aspirantes a soldados, con corte anual, correspondiente al primer y segundo año militar, 
y rendimiento general, así como el nivel de deserción estudiantil y actitud militar analizada por la disciplina y espíritu militar.

A continuación, la siguiente fase, busca comparar la relación existente entre los resultados de las pruebas de ingreso psicológicas, académicas y físicas, con el rendimiento académico, así como la deserción estudiantil y actitud militar analizada por la disciplina y espíritu militar por períodos académicos, en el primer año, segundo año militar y el rendimiento general de los aspirantes a soldados.

Finalmente, en la última fase, se pretende encontrar la prueba de ingreso que predice el rendimiento académico de los aspirantes a soldados, promoción 2018 - 2020.

La recolección de información de las variables se la realiza con la búsqueda en la base de datos del departamento académico de la Escuela, se procede a realizar en una matriz en Excel previamente desarrollada, para luego analizar y correlacionar estadísticamente las variables utilizando el programa estadístico SPSS versión 26, respectivamente. Las variables se expresan como media \pm DE y estas se compararon estadísticamente mediante rho Spearman y regresión lineal.

\section{RESULTADOS}

Pruebas de ingreso que presentaron los aspirantes a soldados.

Se analizó 648 aspirantes de segundo año militar, promoción 2018-2020, de la Escuela de Formación de Soldados del Ejército, los cuales registran en las pruebas de ingreso una media en la prueba académica de 10,03 puntos, seguida de 14,5 puntos en la prueba física, y en lo correspondiente a la prueba psicológica, está dada según el siguiente detalle: en la prueba de capacidad mental una media de B2 y en el test de personalidad B2 igualmente (tabla 1).

Tabla 1. Frecuencia de variables independientes

\begin{tabular}{|c|c|c|c|c|c|c|c|}
\hline & \multicolumn{2}{|c|}{ N } & \multirow[b]{2}{*}{ Media } & \multirow{2}{*}{$\begin{array}{c}\text { Error } \\
\text { estándar de } \\
\text { la media }\end{array}$} & \multirow{2}{*}{$\begin{array}{c}\text { Desv. } \\
\text { Desviación }\end{array}$} & \multirow[b]{2}{*}{ Mínimo } & \multirow[b]{2}{*}{ Máximo } \\
\hline & Válido & Perdidos & & & & & \\
\hline $\begin{array}{l}\text { PRUEBA ACADEMICA DE } \\
\text { INGRESO }\end{array}$ & 619 & 0 & 10,055509 &, 0684348 & 1,7026375 & 5,1200 & 15,6000 \\
\hline $\begin{array}{l}\text { PRUEBA MENTAL DE } \\
\text { INGRESO }\end{array}$ & 619 & 0 & 2,94 &, 035 & 879 & 1 & 4 \\
\hline $\begin{array}{l}\text { PRUEBA DE } \\
\text { PERSONALIDAD DE } \\
\text { INGRESO }\end{array}$ & 619 & 0 & 3,11 &, 021 &, 519 & 1 & 4 \\
\hline $\begin{array}{l}\text { PRUEBA FISICA DE } \\
\text { INGRESO }\end{array}$ & 619 & 0 & 14,634596 & , 1588841 & 3,9529900 & 1,4740 & 20,0000 \\
\hline
\end{tabular}

Fuente: Departamento académico de la Escuela de Formación de Soldados del Ejército "Vencedores del Cenepa"

\section{Rendimiento académico de los aspirantes a soldados}

Del total de aspirantes que ingresaron al Instituto de Formación Militar, 619 (95,5\%) culminaron la carrera de soldados del Ejército, así como la tecnología superior en ciencias 
militares y 29 (4,5\%) desertaron, mismos se detallan a continuación: a) al término del primer período académico desertaron 14 aspirantes como baja voluntaria, b) 9 en el segundo período como baja académica, y c) 1 en el cuarto período y 5 en el quinto período como baja disciplinaria respectivamente (tablas 2 y 3 ).

Tabla 2. Frecuencia de deserción estudiantil

\begin{tabular}{ll|r|r|r|r} 
& Frecuencia & Porcentaje & $\begin{array}{c}\text { Porcentaje } \\
\text { válido }\end{array}$ & $\begin{array}{c}\text { Porcentaje } \\
\text { acumulado }\end{array}$ \\
\hline \multirow{3}{*}{ Válido } & ASPIRANTE & 619 & 95,5 & 95,5 & 95,5 \\
\cline { 2 - 7 } & BAJA ACADÉMICA & 9 & 1,4 & 1,4 & 96,9 \\
\cline { 2 - 7 } & BAJA DISCIPLINARIA & 6 &, 9 &, 9 & 97,8 \\
\cline { 2 - 7 } & BAJA VOLUNTARIA & 14 & 2,2 & 2,2 & 100,0 \\
\cline { 2 - 7 } & Total & 648 & 100,0 & 100,0 & \\
\hline
\end{tabular}

Fuente: Departamento académico de la Escuela de Formación de Soldados del Ejército "Vencedores del Cenepa"

La media del rendimiento del I período académico es de 18,17 puntos, así como 17,86 puntos, 17,81 puntos, 18,13 puntos, 17,97 puntos en el II, III, IV y V período académico respectivamente. Además, la media del rendimiento del I año militar es de 17,81 puntos, así como 18,29 puntos en el II año militar, y 18,05 puntos en el rendimiento general (tabla 4).

Tabla 3. Pruebas de ingreso en deserción estudiantil

\begin{tabular}{|c|c|c|c|c|c|c|c|}
\hline & \multicolumn{2}{|c|}{ N } & \multirow[b]{2}{*}{ Media } & \multirow{2}{*}{$\begin{array}{l}\text { Error } \\
\text { estándar de } \\
\text { la media }\end{array}$} & \multirow{2}{*}{$\begin{array}{c}\text { Desv. } \\
\text { Desviación }\end{array}$} & \multirow[b]{2}{*}{ Mínimo } & \multirow[b]{2}{*}{ Máximo } \\
\hline & Válido & Perdidos & & & & & \\
\hline PRU_ACAD & 29 & 0 & 9,5255 & 29471 & 1,58708 & 7,04 & 12,56 \\
\hline PRU_MENTAL & 29 & 0 & 3,21 & .160 & 861 & 2 & 4 \\
\hline PRU_PERS & 29 & 0 & 3,07 & .110 &, 593 & 1 & 4 \\
\hline PRU_FISI & 29 & 0 & 12,3445 & 1,10540 & 5,95274 & 1,46 & 20,00 \\
\hline DESERCIÓN & 29 & 0 & 3,1724 & .16512 & 88918 & 2,00 & 4,00 \\
\hline
\end{tabular}

Fuente: Departamento académico de la Escuela de Formación de Soldados del Ejército "Vencedores del Cenepa"

La actitud militar valorada en la disciplina y espíritu militar que presentan los aspirantes a soldados está dada por una media de 19,19 puntos, y 18,60 puntos respectivamente (tabla 4). 
Tabla 4. Frecuencia de variables dependientes

\begin{tabular}{|c|c|c|c|c|c|c|}
\hline & \multicolumn{2}{|c|}{$\mathrm{N}$} & \multirow[b]{2}{*}{ Media } & \multirow{2}{*}{$\begin{array}{c}\text { Desv. } \\
\text { Desviación }\end{array}$} & \multirow[b]{2}{*}{ Mínimo } & \multirow[b]{2}{*}{ Máximo } \\
\hline & Válido & Perdidos & & & & \\
\hline PROMEDIO PERIODOI & 619 & 0 & 18,169130 &, 3607970 & 17,0271 & 19,0058 \\
\hline PROMEDIO PERIODO II & 619 & 0 & 17,859996 &, 4163273 & 16,5955 & 18,9387 \\
\hline PROMEDIO PERIODO III & 619 & 0 & 17,809308 &, 4139301 & 16,3676 & 18,9260 \\
\hline PROMEDIO PERIODO IV & 619 & 0 & 18,132632 &, 3303145 & 16,7366 & 19,1096 \\
\hline PROMEDIO GENERAL & 619 & 0 & 18,050061 & ,3257091 & 16,7778 & 18,9754 \\
\hline PROMEDIO 1 AÑO & 619 & 0 & 17,8093 & .41393 & 16,37 & 18,93 \\
\hline PROMEDIO 2 AÑO & 619 & 0 & 18,2908 &, 27846 & 17,19 & 19,09 \\
\hline PROMEDIO PERIODO V & 619 & 0 & 17,970970 &, 3471103 & 16,6153 & 18,9223 \\
\hline DISCIPLINA MIL & 619 & 0 & 19,1875 & .54556 & 16,20 & 20,00 \\
\hline ESPIRITU MIL & 619 & 0 & 18,5980 & ,37073 & 17,51 & 19,43 \\
\hline
\end{tabular}

Fuente: Departamento académico de la Escuela de Formación de Soldados del Ejército "Vencedores del Cenepa"

Relación entre los resultados de las pruebas de ingreso y el rendimiento académico de los aspirantes a soldados

Al realizar el análisis de las comparaciones múltiples, existen diferencias estadísticamente significativas entre la prueba académica y física de ingreso con los rendimientos académicos de los aspirantes a soldados, así como no se evidencia diferencia significativa entre la prueba psicológica con el rendimiento académico correspondiente (tabla 5).

En los cortes anuales del rendimiento académico, así como en el rendimiento general de los aspirantes a soldados existe una correlación directa moderada entre el rendimiento del primer año militar con las pruebas académicas de ingreso $(\mathrm{F}=0,531, \mathrm{p}<0,05)$ y una correlación directa baja con las pruebas físicas de ingreso $(\mathrm{F}=0,279, \mathrm{p}<0,05)$. Una correlación directa baja y significativa entre el rendimiento del segundo año militar con las pruebas académicas de ingreso $(\mathrm{F}=0,393, \mathrm{p}<0,05)$ y con las pruebas físicas de ingreso $(\mathrm{F}=0,264$, $\mathrm{p}<0,05)$. Además, una correlación directa moderada y significativa entre el rendimiento general con las pruebas académicas de ingreso $(\mathrm{F}=0,514, \mathrm{p}<0,05)$ y una correlación directa baja con las pruebas físicas de ingreso $(\mathrm{F}=0,293, \mathrm{p}<0,05)$. Finalmente, no presenta una correlación entre la prueba psicológica (mental y personalidad) con el rendimiento académico correspondiente.

Existe una correlación directa moderada y significativa entre el rendimiento de los 5 períodos académicos con las pruebas académicas de ingreso $(\mathrm{F}=0,413,0,526,0,531,0,412$, 0,516, p $<0,05$ respectivamente) y una correlación directa baja y significativa con las pruebas físicas de ingreso $(\mathrm{F}=0,207,0,267,0,279,0,288,0,305, \mathrm{p}<0,05$ respectivamente), así como no presenta una correlación entre la prueba psicológica (mental y personalidad) con el rendimiento académico correspondiente.

En referencia a la correlación entre las pruebas de ingreso y actitud militar de los aspirantes a soldados, presenta una correlación baja y significativa entre la prueba académica y física de ingreso con la disciplina $(\mathrm{F}=0,140,0,188, \mathrm{p}<0,05)$, así como no correlacionarse con el espíritu militar ( $\mathrm{p}>0,05)$. 
Al correlacionar las pruebas de ingreso con la deserción estudiantil, se evidencia que no existe una correlación significativa con la prueba académica y física de ingreso ( $\mathrm{p}:>0,05)$, pero existe una correlación indirecta moderada y significativa con la capacidad mental de la prueba psicológica $(\mathrm{F}:-0,468, \mathrm{p}:<0,05)$.

\section{Pruebas de ingreso predictivas del rendimiento académico de los aspirantes a soldados}

Las pruebas de ingreso académicas y físicas predicen de forma moderada el rendimiento académico de los aspirantes a soldados y una predicción baja la actitud militar de los aspirantes. Además, se observa que la prueba mental de la prueba psicológica de ingreso predice moderadamente la deserción estudiantil (tabla 6).

Las pruebas de ingreso académicas y físicas predicen el rendimiento académico de primer año militar de forma moderada $\left(\mathrm{R}^{2}: 0,320, \mathrm{p}:<0,05\right)$, así mismo las pruebas de ingreso académicas y físicas predicen el rendimiento académico de segundo año militar de forma moderada $\left(\mathrm{R}^{2}: 0,205, \mathrm{p}:<0,05\right)$, y finalmente las pruebas de ingreso académicas y físicas predicen el rendimiento académico general de forma moderada $\left(\mathrm{R}^{2}: 0,303, \mathrm{p}:<0,05\right)$, la variable de prueba psicológica presenta p: $>0,05$, por lo que es excluida.

Las pruebas de ingreso académicas y físicas predicen el rendimiento de los 5 períodos académicos de forma moderada, observándose en el I período académico un valor $\mathrm{R}^{2}: 0,435$ y $\mathrm{p}:<0,05$, en el II período académico un valor $\mathrm{R}^{2}: 0,560$ y $\mathrm{p}:<0,05$, en el III período académico un valor $\mathrm{R}^{2}$ : 0,565 y p: $<0,05$, en el IV período académico un valor $\mathrm{R}^{2}: 0,454 \mathrm{y}$ $\mathrm{p}:<0,05$ y en el $\mathrm{V}$ período académico un valor $\mathrm{R}^{2}: 0,550 \mathrm{y} \mathrm{p}:<0,05$, finalmente, la variable de prueba psicológica presenta p: $>0,05$, por lo que es excluida.

Las pruebas físicas y académicas de ingreso predicen de forma baja la actitud militar, observándose que para el espíritu militar un valor $\mathrm{R}^{2}: 0,12$ y $\mathrm{p}:<0,05$, y para la disciplina un valor $\mathrm{R}^{2}: 0,065$ y $\mathrm{p}:<0,05$, las variables de pruebas psicológicas presentan $\mathrm{p}:>0,05$, por lo que son excluidas.

La prueba mental de la prueba psicológica de ingreso predice de forma moderada la deserción estudiantil, observándose un valor $\mathrm{R}^{2}: 0,219$ y p: $<0,05$, las variables de pruebas académicas y físicas presentan un p: $>0,05$, por lo que son excluidas. 
Tabla 5. Correlación pruebas de ingreso y rendimiento académico, deserción estudiantil y actitud militar

\begin{tabular}{|c|c|c|c|c|c|c|c|}
\hline & & & $\begin{array}{l}\text { PROMEDIO } \\
\text { PERIODOOI }\end{array}$ & $\begin{array}{c}\text { PRUEBA } \\
\text { ACADEMICA } \\
\text { DE INGRESO }\end{array}$ & $\begin{array}{l}\text { PRUEBA } \\
\text { MENTAL DE } \\
\text { INGRESO }\end{array}$ & $\begin{array}{c}\text { PRUEBA DE } \\
\text { PERSONALID } \\
\text { AD DE } \\
\text { INGRESO }\end{array}$ & $\begin{array}{l}\text { PRUEBA } \\
\text { FISICADE } \\
\text { INGRESO }\end{array}$ \\
\hline \multirow[t]{2}{*}{ Rho de Spearman } & \multirow[t]{2}{*}{ PROMEDIO PERIODOI } & $\begin{array}{l}\text { Coeficiente de } \\
\text { correlación }\end{array}$ & 1,000 & $.413^{\mathrm{m}}$ &,- 062 &,- 054 & $207^{* *}$ \\
\hline & & Sig. (bilateral) & & ,000 & 123 & , 180 & ,000 \\
\hline \multirow[t]{2}{*}{ Rho de Spearman } & \multirow[t]{2}{*}{ PROMEDIO PERIODO ॥ } & $\begin{array}{l}\text { Coeficiente de } \\
\text { correlación }\end{array}$ & 1,000 & $.526^{\mathrm{kn}}$ &,- 064 &,$- 090^{*}$ & $267^{* *}$ \\
\hline & & Sig. (bilateral) & & ,000 &, 112 & , 025 & ,000 \\
\hline \multirow[t]{2}{*}{ Rho de Spearman } & \multirow[t]{2}{*}{ PROMEDIO PERIODO III } & $\begin{array}{l}\text { Coeficiente de } \\
\text { correlación }\end{array}$ & 1,000 & $.531^{\prime \prime}$ &,- 069 &,$- 097^{*}$ & ,279"' \\
\hline & & Sig. (bilateral) & . &, 000 &, 088 & .016 & .000 \\
\hline \multirow[t]{2}{*}{ Rho de Spearman } & \multirow[t]{2}{*}{ PROMEDIO PERIODO IV } & $\begin{array}{l}\text { Coeficiente de } \\
\text { correlación }\end{array}$ & 1,000 & $.412^{\mathrm{m}}$ &,- 046 &,- 068 & $288^{* \prime \prime}$ \\
\hline & & Sig. (bilateral) & & .000 & ,251 & .090 & , 000 \\
\hline \multirow[t]{2}{*}{ Rho de Spearman } & \multirow[t]{2}{*}{ PROMEDIO PERIODO V } & $\begin{array}{l}\text { Coeficiente de } \\
\text { correlación }\end{array}$ & 1,000 & $.516^{\mathrm{m}}$ &,- 060 &,$- 088^{*}$ & $305^{\mathrm{km}}$ \\
\hline & & Sig. (bilateral) & &, 000 &, 133 & .028 &, 000 \\
\hline \multirow[t]{2}{*}{ Rho de Spearman } & \multirow[t]{2}{*}{ PROMEDIO 1 AÑO } & $\begin{array}{l}\text { Coeficiente de } \\
\text { correlación }\end{array}$ & 1,000 & $.531^{\mathrm{N}}$ &,- 069 &,$- 097^{\star}$ & $279^{\mathrm{m}}$ \\
\hline & & Sig. (bilateral) & t &, 000 &, 088 &, 016 &, 000 \\
\hline \multirow[t]{2}{*}{ Rho de Spearman } & \multirow[t]{2}{*}{ PROMEDIO 2 AÑO } & $\begin{array}{l}\text { Coeficiente de } \\
\text { correlación }\end{array}$ & 1,000 &, $393^{\mathrm{m}}$ &,- 038 &,- 052 & $264^{\prime \prime}$ \\
\hline & & Sig. (bilateral) & . &, 000 &, 347 &, 195 &, 000 \\
\hline \multirow[t]{2}{*}{ Rho de Spearman } & \multirow[t]{2}{*}{ PROMEDIO GENERAL } & $\begin{array}{l}\text { Coeficiente de } \\
\text { correlación }\end{array}$ & 1,000 & $.514^{\mathrm{nn}}$ &,- 059 &,$- 087^{*}$ & $293^{\prime \prime}$ \\
\hline & & Sig. (bilateral) & . & ,000 & 143 &, 030 & ,000 \\
\hline \multirow[t]{2}{*}{ Rho de Spearman } & \multirow[t]{2}{*}{ DISCIPLINA MIL } & $\begin{array}{l}\text { Coeficiente de } \\
\text { correlación }\end{array}$ & 1,000 & $.140^{\mathrm{k \prime}}$ &,- 043 &, 002 &, $188^{* \prime \prime}$ \\
\hline & & Sig. (bilateral) & . &, 000 &, 284 &, 958 & .000 \\
\hline \multirow[t]{2}{*}{ Rho de Spearman } & \multirow[t]{2}{*}{ ESPIRITU MIL } & $\begin{array}{l}\text { Coeficiente de } \\
\text { correlación }\end{array}$ & 1,000 & ,079 &,- 015 &,- 026 & .054 \\
\hline & & Sig. (bilateral) & &, 050 &, 707 & .523 & .176 \\
\hline \multirow[t]{2}{*}{ Rho de Spearman } & \multirow[t]{2}{*}{ DESERCIÓN } & $\begin{array}{l}\text { eficiente de } \\
\text { relación }\end{array}$ & 1,000 &, 353 &,$- 488^{\star *}$ &, 124 &, 228 \\
\hline & & . (bilateral) & & ,060 & ,007 & ,521 & ,233 \\
\hline
\end{tabular}

**. La correlación es significativa en el nivel 0,01 (bilateral).

*. La correlación es significativa en el nivel 0,05 (bilateral).

Fuente: Departamento académico de la Escuela de Formación de Soldados del Ejército "Vencedores del Cenepa" 
Tabla 6. Pruebas de ingreso que predicen el rendimiento académico, deserción estudiantil y actitud militar

\begin{tabular}{|c|c|c|c|c|c|c|c|c|c|}
\hline \multirow[b]{2}{*}{ Modelo } & \multirow[b]{2}{*}{$\mathrm{R}$} & \multirow[b]{2}{*}{ R cuadrado } & \multirow[b]{2}{*}{$\begin{array}{c}\text { R cuadrado } \\
\text { ajustado }\end{array}$} & \multirow{2}{*}{$\begin{array}{c}\text { Error } \\
\text { estándar de } \\
\text { la estimación }\end{array}$} & \multicolumn{5}{|c|}{ Estadísticos de cambio } \\
\hline & & & & & $\begin{array}{l}\text { Cambio en R } \\
\text { cuadrado }\end{array}$ & Cambio en $\mathrm{F}$ & gl1 & $\mathrm{g} / 2$ & $\begin{array}{l}\text { Sig. Cambio } \\
\text { en } \mathrm{F}\end{array}$ \\
\hline 1 & $539^{\mathrm{a}}$ & .291 &, 289 & 34891 &, 291 & 252,789 & 1 & 617 &, 000 \\
\hline 2 &, $565^{\mathrm{b}}$ & 320 &, 318 & 34195 & 029 & 26,387 & 1 & 616 &, 000 \\
\hline \multicolumn{10}{|c|}{ *Variable dependiente: Promedio de I año militar } \\
\hline 1 & $397^{\mathrm{a}}$ &, 158 &, 156 &, 25576 &, 158 & 115,580 & 1 & 617 &, 000 \\
\hline 2 & $.452^{\mathrm{b}}$ & 205 & 202 & 24876 &, 047 & 36,208 & 1 & 616 &, 000 \\
\hline \multicolumn{10}{|c|}{ *Variable dependiente: II año militar } \\
\hline 1 & $.512^{\mathrm{a}}$ &, 263 & 261 & .2799373 & .263 & 219,617 & 1 & 617 &, 000 \\
\hline 2 &, $550^{\mathrm{b}}$ & 303 & 301 &, 2723910 &, 040 & 35,660 & 1 & 616 &, 000 \\
\hline \multicolumn{10}{|c|}{ *Variable dependiente: Promedio general. } \\
\hline 1 &, $421^{\mathrm{a}}$ & .177 & .176 &, 3275304 &, 177 & 132,913 & 1 & 617 &, 000 \\
\hline 2 & $.435^{\mathrm{b}}$ &, 189 &, 187 & .3253640 & .012 & 9,244 & 1 & 616 &, 002 \\
\hline \multicolumn{10}{|c|}{ *Variable dependiente: I período académico. } \\
\hline 1 &, $539^{\mathrm{a}}$ & .291 & 289 &, 3509510 & 291 & 252,692 & 1 & 617 &, 000 \\
\hline 2 & $.560^{\mathrm{b}}$ & ,313 &, 311 & 3456008 & .023 & 20,251 & 1 & 616 &, 000 \\
\hline \multicolumn{10}{|c|}{ *Variable dependiente: Il período académico. } \\
\hline 1 & $.539^{\mathrm{a}}$ & ,291 & .289 &, 3489108 & .291 & 252,788 & 1 & 617 &, 000 \\
\hline 2 &, $565^{\mathrm{b}}$ &, 320 &, 318 &, 3419472 &, 029 & 26,386 & 1 & 616 &, 000 \\
\hline \multicolumn{10}{|c|}{ *Variable dependiente: III período académico. } \\
\hline 1 &, $390^{\mathrm{a}}$ &, 152 &, 151 &, 3043585 &, 152 & 110,902 & 1 & 617 &, 000 \\
\hline 2 & $454^{\mathrm{b}}$ &, 206 & 204 &, 2947317 &, 054 & 41,964 & 1 & 616 &, 000 \\
\hline \multicolumn{10}{|c|}{ "Variable dependiente: IV período académico. } \\
\hline 1 &, $507^{\mathrm{a}}$ & .257 &, 256 & 2993995 &, 257 & 213,657 & 1 & 617 &, 000 \\
\hline 2 & $.550^{\mathrm{b}}$ &, 302 &, 300 & ,2903995 &, 045 & 39,836 & 1 & 616 &, 000 \\
\hline
\end{tabular}

a. Predictores: (Constante), PRUEBA ACADEMICA DE INGRESO

b. Predictores: (Constante), PRUEBA ACADEMICA DE INGRESO, PRUEBA FISICA DE INGRESO

\begin{tabular}{|c|c|c|c|c|c|c|c|c|c|}
\hline 1 & $108^{a}$ &, 012 & ,010 & ,36885 &, 012 & 7,324 & 1 & 617 &, 007 \\
\hline \multicolumn{10}{|c|}{$\begin{array}{l}\text { *Variable dependiente: Espíritu militar } \\
\text { a. Predictores: (Constante), PRUEBA ACADEMICA DE INGRESO }\end{array}$} \\
\hline 1 & $234^{\mathrm{a}}$ &, 055 &, 053 & ,53088 &, 055 & 35,650 & 1 & 617 &, 000 \\
\hline 2 & $256^{\mathrm{b}}$ &, 065 &, 062 & 52830 & 011 & 7,040 & 1 & 616 &, 008 \\
\hline
\end{tabular}

*Variable dependiente: Disciplina.

a. Predictores: (Constante), PRUEBA FISICA DE INGRESO

b. Predictores: (Constante), PRUEBA FISICA DE INGRESO, PRUEBA ACADEMICA DE INGRESO

\begin{tabular}{lccccccccc}
\hline 1 & $.468^{\text {a }}$ &, 219 &, 190 &, 80017 &, 219 & 7,576 & 1 & 27 &, 010 \\
\hline *Variable dependiente: Deserción estudiantil. & & & & & & &
\end{tabular}

a. Predictores: (Constante), PRU_MENTAL

Fuente: Departamento académico de la Escuela de Formación de Soldados del Ejército "Vencedores del Cenepa" 


\section{DISCUSIÓN}

\section{Pruebas de ingreso}

No existen estudios suficientes sobre la relación de las pruebas de ingreso y rendimiento académico en el contexto de instituciones educativas militares, por ello el análisis del estudio se los realiza con los pares en instituciones educativas universitarias regulares.

Los resultados de las pruebas de ingreso en estudios similares son variados, no existe una tendencia establecida que nos pueda indicar el corte adecuado de ingreso a Institutos Universitarios.

Se observa que la media del promedio de la prueba académica de ingreso de los aspirantes a soldados es similar a un estudio en 116 estudiantes de la carrera de medicina de una universidad del norte de México, en donde el promedio de la prueba académica de ingreso es de 50,8/100 puntos (equivalencia de 10,16/20 puntos) (Gómez-López et al, 2012). Situación similar a lo observado en 572 estudiantes del grado de finanzas y contabilidad de la Universidad de Sevilla en España, en donde la media de la nota de acceso de 5,804/10 puntos (equivalencia de 11,61/20 puntos) (Jiménez-Caballero et al, 2015). Contrario a lo que sucede en un estudio en 130 alumnos de la escuela de medicina de la Universidad de Azuay en Ecuador que presentan una media de la prueba de ingreso de 79,20/100 puntos (equivalente a 15,84/20 puntos) (Alvear, 2014).

Llama la atención que la nota mínima de ingreso al instituto de formación militar sea de 5,12/20 puntos, así como la nota máxima de ingreso de 15,5/20 puntos, lo cual pone de manifiesto que es un grupo con conocimientos y habilidades muy heterogéneos y definidos como rendimientos regulares - deficientes (COMACO, 2016).

Al ser los institutos de formación militar de los pocos centros universitarios que en el perfil de ingreso el aspirante tiene que ser apto físicamente, no existen estudios similares en los que las pruebas físicas sean parte del proceso de selección. Pese a ello, se observa una media de pruebas físicas de ingreso regulares, de 14,53/20 puntos, con una nota mínima de 1,46/20 puntos (deficiente), lo cual demuestra un déficit en la preparación física previa de los aspirantes a soldados (COMACO, 2016).

El análisis de los resultados de la prueba psicológica de ingreso, evidencia una media en la prueba mental de B2 y en la prueba de personalidad B2 igualmente, con un ingreso de hasta B3, lo cual según el perfil de ingreso no es recomendable. En los aspirantes ingresados las capacidades mentales y perfil de personalidad están alejadas de lo ideal, resultado que predispone a un alto riesgo de fracaso (CEDE, 2011).

\section{Rendimiento académico}

La media del promedio al finalizar el primer año militar en los aspirantes a soldados es de 17,81/20 puntos, la cual está ligeramente inferior a lo observado en un estudio similar en estudiantes de medicina de la Universidad de Azuay en Ecuador, que se encuentra en 18,23/20 puntos (Alvear, 2014). Así mismo, superior a lo que sucede en la Universidad Veracruzana de México, en donde se observa un promedio de promoción de 77,24/100 puntos (equivalencia de 15,45/20 puntos) (Chain et al, 2003), y a lo observado en el grado de finanzas y contabilidad de la Universidad de Sevilla en España, en donde la media del 
promedio en las asignaturas es de 4,04/10 puntos (equivalencia de 8,08/20 puntos) (JiménezCaballero et al, 2015). Además, es necesario destacar que no existe gran variación del rendimiento académico mínimos y máximos (16,37/20 puntos y 18,93/20 puntos respectivamente) de los aspirantes a soldados.

En el instituto de formación militar el porcentaje de deserción estudiantil es bajo $(4,5 \%)$ en comparación al existente en otros centros universitarios regulares, encontrándose un $88,2 \%$ de deserción de estudiantil en la carrera de arquitectura y diseño gráfico de una Universidad de Guatemala durante el período 2011 - 2018 (Rabe, 2019) y lo observado en estudiantes de medicina de la universidad de Azuay en Ecuador, que presenta una deserción estudiantil del 30,76\% (Alvear, 2014).

Analizando por períodos académicos a los aspirantes a soldados, se puede apreciar que se mantiene cierta tendencia en el desarrollo del rendimiento académico durante los dos años de carrera, presentando un intervalo de medias entre 16,37/20 puntos en el III período a 19,10/20 puntos en el I período académico.

Los aspirantes a soldados al finalizar su segundo año de formación militar presentan una media del promedio de 18,29/20 puntos, el cual es superior al promedio observado en estudiantes al finalizar la carrera de medicina de una universidad del norte de México de 80,0/100 (equivalencia de 16,00/20 puntos) (Gómez-López et al, 2012).

La actitud militar que presentan los aspirantes durante su permanencia en los 2 años de carrera demuestra una media del promedio excelente y bueno para disciplina y espíritu militar $(19,19 / 20$ puntos y 18,60 puntos respectivamente), pero existe una diferencia marcada en los extremos de los mismos, promedio mínimo de 16,20/20 puntos, máximo de 20/20 puntos en disciplina, y un promedio mínimo de 17,51/20 puntos y máximo de 19,43/20 puntos para espíritu militar, lo que demuestra que es un grupo estudiantil con actitud militar variable y heterogéneo.

\section{Correlación entre pruebas de ingreso y rendimiento académico}

Al realizar el análisis relacional se puede evidenciar una correlación directa moderada y baja entre los resultados de las pruebas académicas y físicas de ingreso a los institutos de formación militar con el rendimiento académico, deserción estudiantil y actitud militar de los aspirantes a soldados, situación contraria se observa con el resultado de la prueba psicológica, la cual no presentan una correlación significativa. Lo que pone de manifiesto que los resultados de las pruebas de ingreso, más acentuado con la prueba psicológica, no cumplen la función de admitir a los mejores aspirantes para su formación como soldados, hecho observable en el perfil psicológico de ingreso mínimo: B3 en el test de personalidad y B3 en capacidad mental, así como promedios en las pruebas académicas de ingreso mínimas: 5,12/20 puntos y promedios en las pruebas físicas de ingreso mínimas: 1,46/20 puntos admitidos para ingresar al instituto de formación militar.

La correlación de las pruebas académicas de ingreso con el rendimiento académico de los aspirantes a soldados en el primer año militar ( $\mathrm{F}: 0,539, \mathrm{p}:<0,05), \mathrm{p}:<0,05)$ y promedio general $(\mathrm{F}: 0,512, \mathrm{p}:<0,05)$ evidencia una correlación directa moderada y una correlación directa baja con el segundo año militar (F: 0,397, p:<0,05), similar a la correlación observada en 572 estudiantes del grado de finanzas y contabilidad de la Universidad de Sevilla en 
España (F: 0,439) (Jiménez-Caballero et al., 2015), en contraste a lo observado en un estudio en la Universidad Técnica de Ambato en Ecuador en donde la correlación es baja (R: 0,13 y R: 0,21) (López-Altamirano et al, 2020), así como lo observado en un estudio en la universidad de Guatemala, en donde las pruebas de ingreso no se relacionan positivamente con el rendimiento académico de sus alumnos (R: 0,47 para primer semestre y R: -0,35 para segundo semestre) (Rabe, 2019). La correlación de las pruebas físicas y rendimiento académico en los aspirantes a soldados igualmente es moderada y significativa, hecho no observable con la prueba psicológica, la cual no presenta correlación.

La correlación de la deserción estudiantil con las pruebas de ingreso de los aspirantes a soldados no evidencia una correlación significativa, misma se detalla a continuación: no existe correlación significativa con las pruebas académicas y físicas (p:>0,05), una correlación indirecta moderada y significativa con la capacidad mental de la prueba psicológica de ingreso ( $\mathrm{F}$ : $-0,468, \mathrm{p}:<0,05)$. Además, es importante denotar que la media de las pruebas de ingreso que presentaron los aspirantes que desertaron (prueba académica: 9,53/20 puntos, prueba física: 12,35/20 puntos y prueba psicológica: B2) es similar al que presentaron los aspirantes en general (prueba académica: 10,03/20 puntos, prueba física: 14,53/20 puntos y prueba psicológica: B2).

Situación similar ocurre entre las pruebas de ingreso y actitud militar, en donde no existe una correlación significativa adecuada entre las mismas.

\section{Predictores del rendimiento académico}

Las pruebas de ingreso que predicen el rendimiento académico y actitud militar de los aspirantes a soldados son las pruebas académicas y físicas, se excluye a los resultados de la prueba psicológica, excepto a la prueba mental que es la única variable de la prueba psicológica que predice el rendimiento académico.

El 32\% de la variación del rendimiento académico de primer año militar está explicado por las pruebas de ingreso académicas y físicas $\left(\mathrm{R}^{2}: 0,320, \mathrm{p}:<0,05\right)$, así mismo el $21 \%$ de las variación del rendimiento académico de segundo año militar está explicado por las pruebas de ingreso académicas y físicas $\left(\mathrm{R}^{2}: 0,205, \mathrm{p}:<0,05\right)$ y finalmente el $30 \%$ de las variación del rendimiento académico general de los aspirantes está explicado por las pruebas de ingreso académicas y físicas $\left(\mathrm{R}^{2}: 0,303\right.$, p:<0,05), en contraste al 19,3\% de la variación en las asignaturas del Grado de Finanzas y Contabilidad de la Universidad de Sevilla en España es explicada por la nota de ingreso (Jiménez-Caballero et al., 2015). La variable de la prueba psicológica presenta p: >0,05, por lo que es excluida del cuadro de predicción.

El $44 \%$ de la variación del rendimiento académico en el I período académico está explicado por las pruebas de ingreso académicas y físicas $\left(\mathrm{R}^{2}: 0,435, \mathrm{p}:<0,05\right)$, así como el $56 \%$ de la variación en el II período académico $\left(\mathrm{R}^{2}: 0,560, \mathrm{p}:<0,05\right)$, el $57 \%$ de la variación en el III período académico $\left(\mathrm{R}^{2}: 0,560, \mathrm{p}:<0,05\right)$, el $45 \%$ de la variación en el IV período académico $\left(\mathrm{R}^{2}: 0,560, \mathrm{p}:<0,05\right)$ y finalmente el $55 \%$ de las variación del rendimiento académico en el $\mathrm{V}$ período académico (R2: 0,550, p:<0,05), la variable prueba psicológica presenta p: $>0,05$, por lo que es excluida del cuadro de predicción.

En relación a la actitud militar, se tiene que el $12 \%$ de la variación del espíritu militar está explicado por las pruebas de ingreso académicas y físicas (R2: 0,12, p:<0,05), así como el 
6\% de la variación de la disciplina (R2: 0,065, p:<0,05), la variable prueba psicológica presenta p: >0,05, por lo que es excluida del cuadro de predicción.

La prueba psicológica de ingreso predice de forma moderada la deserción estudiantil, observándose que el 22\% de la variación de la deserción estudiantil está explicado por la prueba mental (R2: 0,219 y p: <0,05), las variables de pruebas académicas y físicas presentan un p: $>0,05$, por lo que son excluidas.

\section{Limitaciones}

La falta de estudios previos en el contexto educativo militar sobre pruebas de ingreso, rendimiento académico y su relación, pone de manifiesto una evidente limitación en el desarrollo del presente trabajo investigativo, mismo que sirve como una oportunidad para identificar nuevas brechas en la literatura educativa y consecuentemente nuevas investigaciones en el contexto militar.

\section{Replicabilidad}

En Ecuador existen tres institutos de formación militar pertenecientes al Ejército, Escuela Superior Militar "Eloy Alfaro", Escuela de Formación de Soldados del Ejército "Vencedores del Cenepa" y Escuela de IWIAS “Tcrn. Gonzalo Barragán”, los cuales tienen diferente misión y visión, enmarcados en los requerimientos de competencias particulares que desea la Institución Militar para su personal militar. Situación que permite replicar el presente trabajo investigativo con el fin de conocer la realidad de la predicción las pruebas de ingreso en la admisión de aspirantes y/o cadetes con conocimientos y habilidades específicas, y la efectividad de los perfiles de ingreso actuales de cada escuela, establecidos por el sistema educativo militar.

\section{CONCLUSIÓN}

Las pruebas de ingreso que se aplican en el período de admisión a los institutos de formación militar no presentan una predicción fuerte del rendimiento académico, deserción estudiantil y actitud militar de los aspirantes a soldados durante el desarrollo de la Carrera de Tecnología Superior en Ciencias Militares. Ello implica plantear la formulación de un perfil de ingreso nuevo para contar con estándares de calidad acordes al contexto, que permita la admisión de aspirantes con conocimientos y habilidades óptimos para cumplir las exigencias académico militares y actitudinales propias de la formación como soldados, y estén en la capacidad de enfrentar los nuevos retos en la seguridad y defensa del estado en el futuro.

Los resultados de las pruebas de ingreso de los aspirantes a soldados que fueron admitidos al instituto de formación militar evidencia que se cumple con la demanda de cupos establecidos, pero se permite cierta flexibilidad en la aceptación con resultados que se contraponen al perfil de ingreso deseable en la carrera de tecnología superior en ciencias militares establecidos. Esto predispone a contar con una población estudiantil con conocimientos y habilidades previas heterogéneas, lo que dificulta adaptarse a las exigencias académicas militares y actitudinales propias de un aspirante a soldado. 
El proceso de selección a los institutos de formación militar es un esfuerzo primordial de la Fuerza Terrestre, que pretende elegir a los mejores estudiantes que garanticen el éxito académico y profesional posterior. Sin embargo, los resultados de las pruebas de ingreso no presentan una correlación bien establecida con el rendimiento académico de los aspirantes a soldados de la Escuela de Formación de Soldados del Ejército "Vencedores del Cenepa". Esto abre la oportunidad de motivar el desarrollo de una propuesta metodológica propedéutica, a fin de contar con un grupo estudiantil con conocimientos y habilidades más homogéneas, que permita una calidad de aspirantes a soldados, así como profesionales académica, física y mentalmente mejor preparados.

\section{NOTA SOBRE LOS AUTORES:}

Germán Geovanny Muñoz Gualán. Médico, Maestrante de innovación educativa de la Universidad Católica de Ecuador sede Ambato, Maestrante de gerencia de instituciones de salud de la Universidad Técnica Particular de Loja. Así mismo, es jefe del Centro de Salud "Esforse", Coordinador docente del módulo Sanidad Militar de la Escuela de formación de soldados del Ejército - UFA ESPE. Correo electrónico: gergeomunoz1988@ gmail.com

Efrain Eduardo Zambrano Rosales. Ingeniero de Empresas, Magister en Docencia Universitaria y Currículo para la Educación Superior, Maestrante MBA en la Universidad Técnica de Cotopaxi, Doctorante PhD en Administración de la Universidad Nacional de Rosario, Argentina. Así mismo, es docente universitario Titular Auxiliar, Facilitador del módulo de Logística Inversa y Logística y Cadena de Suministro en Maestría de Transportes de la Escuela Politécnica de Chimborazo. Correo electrónico: e2zr@yahoo.com

\section{REFERENCIAS}

Álvarez, M-Valle y Vallelado, Eleutério (2013), “Algunas Dimensiones relacionadas con el Rendimiento Académico de Estudiantes de Administración y Dirección de Empresas", Universitas psychologica, Vol. 12, No. 3, pp. 739-752.

Alvear, Christopher (2014), Asociación entre indicadores de rendimiento académico y la aprobación del primer año en la Facultad de Medicina, Trabajo de fin de grado. Universidad del Azuay, Quito (Ecuador).

Batallas González, David G. (2014), “Análisis de los factores que determinan el rendimiento académico de los estudiantes universitarios", Revista científica sinapsis, Vol. 1, No. 4.

Castro Solano, Alejandro y Fernández Liporace, Mercedes (2011), "Predictores Para la Selección de Cadetes en Instituciones Militares”, Psykhe, Vol. 14, No. 1, pp. 17-30.

CEDE (2011), Normativa de Asesoría Psicopedagógica, Basada en el Enfoque por Competencias, Sangolquí: Fuerza Terrestre del Ecuador.

Chain Revuelta, Ragueb; Cruz Ramírez, Nicandro; Martínez Morales, Manuel y Jácome Ávila, Nancy (2003), "Examen de Selección y Probabilidad de Éxito Escolar en Estudios Superiores: Estudio en una Universidad Pública Estatal mexicana", Revista electrónica de investigación educativa, Vol. 5, No. 1, pp. 1-17. 
COMACO (2016), Modelo Educativo de las Fuerzas Armadas, Quito: Fuerzas Armadas del Ecuador.

Depaula, Pablo D. (2012), "Predictores Globales de la Performance de Estudiantes Militares", Ciencias Psicológicas, Vol. 6, No. 2, pp. 135-148.

Fernández Aguerre, Tabaré (2004), "Clima Organizacional en las Escuelas: Un Enfoque Comparativo para México y Uruguay", REICE. Revista Iberoamericana sobre Calidad, Eficacia y Cambio en Educación, Vol. 2, No. 2, pp. 43-68.

Garbanzo Vargas, Guiselle M. (2007), "Factores Asociados al Rendimiento Académico en Estudiantes Universitarios, una Reflexión Vista desde la Calidad de la Educación Pública", Revista De Educación, Vol. 31, No. 1, pp. 43-63.

Gómez-López, Victor M.; Rosales-Gracia, Sandra; Marín-Solórzano, Griselda; GarcíaGalaviz, José L. y Guzmán-Acuña, Josefina (2012), "Correlación entre el examen de selección y el rendimiento académico al término de la carrera de Medicina", Educación Médica Superior, Vol. 26, No. 4, pp. 502-513.

Jiménez-Caballero, José L.; Camúñez Ruiz, José A.; González-Rodríguez, M. Rosario y Fuentes Ruiz, Pilar (2015), "Factores determinantes del rendimiento académico universitario en el Espacio Europeo de Educación Superior", Innovar, Vol. 25, No. 58, pp. 159-176.

León Carrera, Edison J. (2009), Construcción y validación de indicadores del aspirante idóneo a una Institución Militar, Trabajo de fin de maestría. Escuela Politécnica Nacional, Quito (Ecuador).

López, Ciro R. (2016), Factores demográficos, socioeconómicos e institucionales que influyen en el rendimiento académico de los estudiantes universitarios, Trabajo de fin de maestría. Universidad Nacional de Córdoba, Río Cuarto Argentina.

López-Altamirano, Diego A.; Gómez-Morales, Mariana de J.; Mayorga-Alvarado, Fanny del R.; Paredes-Ojeda, María E. y Martínez-Pérez, Sonia G. (2020), "La puntuación del examen Ser Bachiller como predictor del rendimiento académico universitario", Polo del Conocimiento, Vol. 5, No. 3, pp. 69-91.

Rabe Rendón, Byron A. (2019), "Las pruebas de ingreso a la Facultad de Arquitectura y su relación con el rendimiento académico: primera parte: fundamentos y resultados para la carrera de Arquitectura", Avance, pp. 1-25.

Rodríguez Fontes, Raúl; Díaz Rodríguez, Pedro A.; Moreno Lazo, Mirna y Bacallao Gallestey, Jorge (2000), "Capacidad Predictiva de varios Indicadores de Selección para el Ingreso a la Carrera de Medicina", Educación Médica Superior, Vol. 14, No. 2, pp. 128-35.

Maquilón Sánchez, Javier J. y Hernández Pina, Fuensanta (2011), "Influencia de la motivación en el rendimiento académico de los estudiantes de formación profesional", Revista electrónica interuniversitaria de formación del profesorado, Vol. 14, No. 1, pp. 81100.

Santos, M. V.; García, M. T. y Vallelado, E. (2010), "La percepción directiva de distintos estímulos estratégicos", Ponencia presentado en el XX Congreso Nacional de la Asociación Científica de Economía y Dirección de la Empresa, Granada (España). 
Universidad de Fuerzas Armadas - ESPE (2017), Proyecto de Rediseño Curricular de la Carrera de Tecnología Superior en Ciencias Militares, Quito: Universidad de Fuerzas Armadas - ESPE.

Zapata Villacís, Luis A. (2017), "Modelo de ingreso a la Universidad Central del Ecuador y rendimiento académico", Revista Científica Retos de la Ciencia, Vol. 1, No. 2, pp. 71-79.

Zwick, Rebecca J. (2012), "The role of admissions test scores, socioeconomic status, and high school grades in predicting college achievement", Pensamiento Educativo, Revista de Investigación Educacional Latinoamericana, Vol. 49, No. 2, pp. 23-30. 